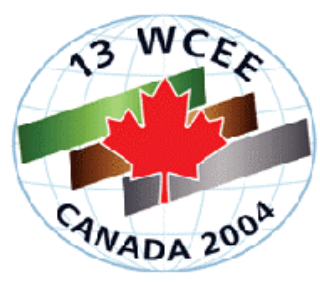

$13^{\text {th }}$ World Conference on Earthquake Engineering

Vancouver, B.C., Canada

August 1-6, 2004

Paper No. 2289

\title{
TORSIONAL SEISMIC RESPONSE CONTROL OF ASYMMETRIC-PLAN SYSTEMS BY USING VISCOUS DAMPERS
}

\author{
Bruno PALAZZO ${ }^{1}$, Luigi PETTI ${ }^{1}$, Massimiliano DE IULIIS ${ }^{1}$
}

\begin{abstract}
SUMMARY
An investigation on the torsional seismic response controlling capacity of dissipation devices in the asymmetric-plan buildings is herein presented. Effects of the plan-wise distribution of supplemental damping on torsionally dynamic behaviour have been investigated by using modal analysis techniques in the state space representation. Parametrical analysis leads to the optimal plan-wise allocation of damping resources on varying the dynamic characteristics of the asymmetric-plan system. Results are also obtained by both applying $\mathrm{H}_{2}$ and $\mathrm{H}_{\infty}$ norm control methods and by numerically analysing the dynamic response to recorded and synthetic seismic excitations.
\end{abstract}

\section{INTRODUCTION}

Studies on the seismic response of asymmetric-plan systems have always aroused considerable interest in the scientific community [Hejal, R. and Chopra, A., 1987] [Kan, C. L. and Chopra, A. K., 1979]. The importance of torsional effects on the seismic behaviour of structures having an irregular plan distribution of mass and stiffness is generally taken into account in aseismic provisions and guidelines for the design of seismic-resistant systems.

From the beginning of the 1990s, assessment studies started to evaluate the possibilities of utilising extrastructural damping in order to reduce seismic demand in asymmetric-plan systems [Pekau OA, Guimond R., 1991], [Goel RK, 1998]. Recent studies have demonstrated the effectiveness of such a control strategy in reducing both the linear [Goel RK, 2000], [Lin W-H, Chopra AK, 2001] and non-linear [Goel RK, Booker A., 2001] seismic response of asymmetric systems through the use of viscous-fluid devices. These studies have pointed out the importance of the plan-wise distribution of additional damping devices by supplying a sort of design guideline. The result of these studies is to arrange the supplemental dampers such that the damping eccentricity respect to the mass centre takes on the largest value with algebraic sign opposite to the structural eccentricity [Goel RK, 2000]. This means locate the supplemental damping centre on the flexible edge side.

This study faces the same problem through methodologies of "vibration control theory". In particular, linear seismic response of non-proportional damped systems is investigated through the use of modal analysis techniques and $\mathrm{H}_{2}$ and $\mathrm{H}_{\infty}$ transfer function norms. Parametrical analyses are carried out for the definition of supplemental damping design criteria by considering the mass and stiffness properties of the

\footnotetext{
${ }^{1}$ Department of Civil Engineering - University of Salerno (Italy)
} 
asymmetric system. Finally, the design criteria have also been tested through the dynamic analysis of asymmetric systems subjected both to synthetic and recorded seismic events.

\section{DYNAMIC ANALYSIS OF ASYMMETRIC-PLAN SYSTEMS EQUIPPED WITH SUPPLEMENTAL DAMPING}

Let us consider the structure shown in figure 1 described in a co-ordinate system where the origin of the axes is located to coincide with the centre of stiffness $C_{K}$ and the direction of $x$-axis is described by the line connecting $C_{K}$ with the centre of masses $C_{M}$. System asymmetry is defined by eccentricity $e$, which is the distance between the centres of mass and stiffness.

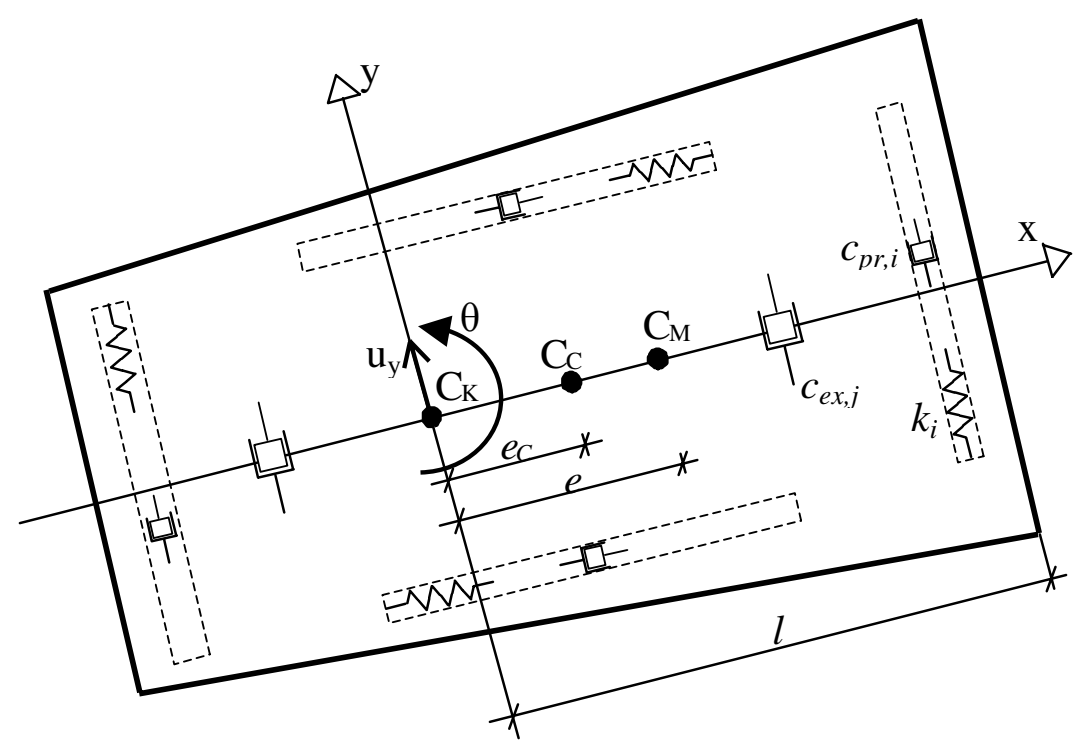

Figure 1: Model of an asymmetric-plan system

The particular choice of this co-ordinate system brings about an uncoupling of translation displacement along the $x$-axis from that which is lateral-torsional along the $y$-axis. In this paper we will examine only the lateral-torsional behaviour as defined by the two degrees of freedom $\left(u_{y}, \theta\right)$.

The system is characterized by its natural damping parameters proportional to its mass and stiffness by means of $\alpha$ and $\beta$, and with extra-structural damping devices described by viscous damping constants $c_{x, i}$ and $c_{y, i}$ respectively in directions $x$ and $y$. The equations of motion are derived for the two degrees of freedom system as:

$$
\left\{\begin{array}{l}
m\left(\ddot{y}+\frac{e}{l}(l \ddot{\theta})\right)+\left[\alpha m\left(\dot{y}+\frac{e}{l}(l \dot{\theta})\right)+\beta k_{y} \dot{y}\right]+c_{y, e x t}\left(\dot{y}+\frac{e_{C}}{l}(l \dot{\theta})\right)+k_{y} y=-m \ddot{u}_{g, y} \\
m\left(\frac{e}{l} \ddot{y}+\frac{\rho_{m}^{2}}{l^{2}}(l \ddot{\theta})\right)+\left[\alpha m\left(e \dot{y}+\frac{\rho_{m}^{2}}{l^{2}}(l \dot{\theta})\right)+\beta k_{y} \frac{\rho_{k}^{2}}{l^{2}}(l \dot{\theta})\right]+c_{y, e x t}\left(e_{C} \dot{y}+\frac{\rho_{C}^{2}}{l^{2}}(l \dot{\theta})\right)+k_{y} \frac{\rho_{k}^{2}}{l^{2}}(l \theta)=-m \frac{e}{l} \ddot{u}_{g, y}
\end{array}\right.
$$


In (1), the supplemental damping system is described by eccentricity $e_{C}$ between the centre of damping $C_{C} \equiv\left(x_{C}, y_{C}\right) \quad$ and the centre of stiffness $C_{K}$, the radius of gyration $\rho_{C}$ and the overall damping coefficient $c_{y, \text { ext }}$ in direction $\mathrm{y}$ :

$$
\begin{array}{cc}
x_{C}=\sum_{i=1}^{n} c_{y, i} x_{i} / \sum_{i=1}^{n} c_{y, i} & y_{C}=\sum_{i=1}^{n} c_{x, i} y_{i} / \sum_{i=1}^{n} c_{x, i} \\
\rho_{C}=\sqrt{\frac{\sum_{i=1}^{n} c_{y, i} x_{i}^{2}+\sum_{i=1}^{n} c_{x, i} y_{i}^{2}}{\sum_{i=1}^{n} c_{y, i}+\sum_{i=1}^{n} c_{x, i}}} \quad c_{y, e x t}=\sum_{i=1}^{n} c_{y, i}
\end{array}
$$

In the following, $l$ indicates the projection along the x-axis of the edge of the system, $n$ is the number of dampers, and finally, $x_{i}$ and $y_{i}$ the coordinates which identify the position of the $i$-nth device. Equations (1) can be written in the form:

$$
\left[\begin{array}{cc}
1 & \frac{e}{l} \\
\frac{e}{l} & \frac{\rho_{m}^{2}}{l^{2}}
\end{array}\right]\left\{\begin{array}{l}
\ddot{y} \\
l \ddot{\theta}
\end{array}\right\}+\left[\alpha\left[\begin{array}{cc}
1 & \frac{e}{l} \\
\frac{e}{l} & \frac{\rho_{m}^{2}}{l^{2}}
\end{array}\right]+\beta \omega_{y}^{2}\left[\begin{array}{cc}
1 & 0 \\
0 & \frac{\rho_{K}^{2}}{l^{2}}
\end{array}\right]\left\{\begin{array}{c}
\dot{y} \\
l \dot{\theta}
\end{array}\right\}+2 \xi_{y} \omega_{y}\left[\begin{array}{cc}
1 & \frac{e_{C}}{l} \\
\frac{e_{C}}{l} & \frac{\rho_{C}^{2}}{l^{2}}
\end{array}\right]\left\{\begin{array}{c}
\dot{y} \\
l \dot{\theta}
\end{array}\right\}+\omega_{y}^{2}\left[\begin{array}{cc}
1 & 0 \\
0 & \frac{\rho_{K}^{2}}{l^{2}}
\end{array}\right]\left\{\begin{array}{c}
y \\
l \theta
\end{array}\right\}=-\left[\begin{array}{cc}
1 & \frac{e}{l} \\
\frac{e}{l} & \frac{\rho_{m}^{2}}{l^{2}}
\end{array}\right]\left\{\begin{array}{c}
1 \\
0
\end{array}\right\} \ddot{u}_{g, y}\right.
$$

where $\xi_{y}$ and $\omega_{y}$ represent the supplemental viscous damping factor and the pure translation natural circular frequency respectively. In a symbolic form (4) can be rewritten as:

$$
\mathbf{M} \ddot{\zeta}+(\alpha \mathbf{M}+\beta \mathbf{K}) \dot{\zeta}+\mathbf{C}_{e x t} \dot{\zeta}+\mathbf{K} \zeta=-\mathbf{M I}_{\mathrm{g}} \ddot{u}_{g}
$$

where $\mathbf{M}, \mathbf{C}_{e x t}$ and $\mathbf{K}$ represent the mass, supplemental damping and stiffness matrices respectively, $\mathbf{I}_{g}$ is the influence vector of the ground motion and $\zeta=\left[\begin{array}{ll}y & l \theta\end{array}\right]^{T}$ the displacement vector.

The supplemental damping may be considered as a control action described by $\mathbf{u}=\mathbf{C} \dot{\zeta}$. Equation (5) can therefore be rewritten in the form:

$$
\mathbf{M} \ddot{\zeta}+(\alpha \mathbf{M}+\beta \mathbf{K}) \dot{\zeta}+\mathbf{K} \zeta=-\mathbf{M I}_{\mathbf{g}} \ddot{u}_{g}-\mathbf{u}
$$

which in state space representation leads to:

$$
\left\{\begin{array}{l}
\dot{\zeta} \\
\ddot{\zeta}
\end{array}\right\}=\left[\begin{array}{cc}
\mathbf{0}_{2 x 2} & \mathbf{I}_{2 x 2} \\
-\mathbf{M}^{-1} \mathbf{K} & -\mathbf{M}^{-1}(\alpha \mathbf{M}+\beta \mathbf{K})
\end{array}\right]\left\{\begin{array}{l}
\boldsymbol{\zeta} \\
\dot{\zeta}
\end{array}\right\}+\left[\begin{array}{c}
\mathbf{0}_{2 x 1} \\
-\mathbf{I}_{\mathbf{g}}
\end{array}\right] \ddot{u}_{g}+\left[\begin{array}{c}
\mathbf{0}_{2 x 2} \\
-\mathbf{M}^{-1}
\end{array}\right] \mathbf{u}
$$

or rather, in symbolic form:

$$
\left\{\begin{array}{l}
\dot{\mathbf{Z}}=\mathbf{A Z}+\mathbf{B}_{\mathbf{g}} \ddot{u}_{g}+\mathbf{B}_{\mathbf{u}} \mathbf{u} \\
\mathbf{u}=\mathbf{K}_{\mathbf{1}} \mathbf{Z}
\end{array}\right.
$$


where $\mathbf{Z}=\left[\begin{array}{ll}\zeta & \dot{\zeta}\end{array}\right]^{T}=\left[\begin{array}{llll}y & l \theta & \dot{y} & l \dot{\theta}\end{array}\right]^{T}$ is the state vector of the system, with $\mathbf{A}$ the state matrix for the uncontrolled structure and with $\mathbf{K}_{1}=\left[\begin{array}{ll}\mathbf{0}_{2 x 2} & \mathbf{C}_{e x t}\end{array}\right]^{T}$ the gain matrix that connects the control action to the system state.

In the complex Laplace space, equation (8) can be written as:

$$
\left\{\begin{array}{l}
s \mathbf{I Z}=\mathbf{A} \mathbf{Z}+\mathbf{B}_{\mathbf{g}} s^{2} U_{g}+\mathbf{B}_{\mathbf{u}} \mathbf{u} \\
\mathbf{u}=\mathbf{K}_{\mathbf{1}} \mathbf{Z}
\end{array}\right.
$$

By defining $\mathbf{H}=(s \mathbf{I}-\mathbf{A})^{-1} \mathbf{B}_{\mathrm{g}} s^{2}$ the transfer matrix relating the complex response of the uncontrolled system to the input seismic action, the system (9) can be rewritten as:

$$
\left\{\begin{array}{l}
\mathbf{Z}=\mathbf{H} U_{g}+\mathbf{H} \frac{\mathbf{B}_{\mathbf{u}}}{\mathbf{B}_{g} s^{2}} \mathbf{u} \\
\mathbf{u}=\mathbf{K}_{\mathbf{1}} \mathbf{Z}
\end{array}\right.
$$

From (10) the control block diagram, shown in fig. 2, which is representative of the behaviour of an asymmetric-plan system equipped with extra-structural damping is obtained [Palazzo B., Petti L., 1997].

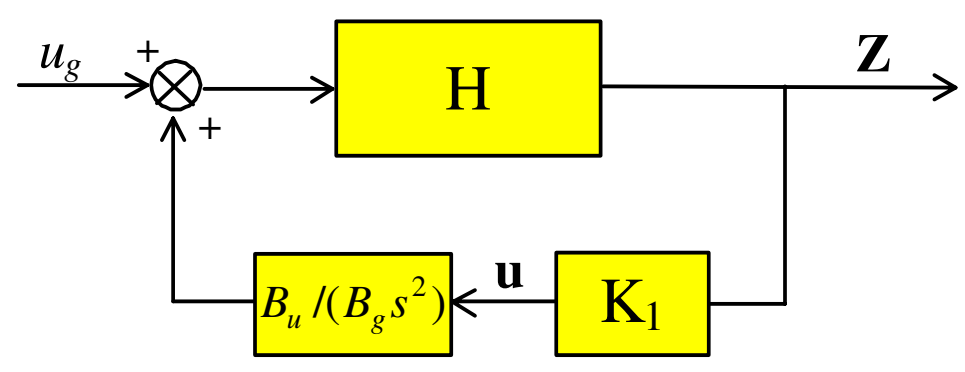

Figure 2: Block diagram of the controlled system

It is interesting to note that the supplemental dissipation is seen by the system as a closed loop controller in which the control action works retroactively on the system. It can be observed that, although the controller input is the complete state of the system, the specific form of the gain matrix, $\mathbf{K}_{1}$, only allows for direct control of the velocity components. Therefore, displacement control is obtained by indirectly controlling the velocity. By means of supplemental energy dissipation, it is therefore possible to control efficiently the relative displacements coupled to high values of velocity components.

System (9) can also be rewritten as:

$$
\dot{\mathbf{Z}}=\left(\mathbf{A}+\mathbf{B}_{\mathbf{u}} \mathbf{K}_{1}\right) \mathbf{Z}+\mathbf{B}_{\mathbf{g}} \ddot{u}_{g}
$$

In this case, $\left(\mathbf{A}+\mathbf{B}_{\mathbf{u}} \mathbf{K}_{\mathbf{1}}\right)$ represents the state matrix of the controlled system. 


\section{MODAL ANALYSIS IN THE SPACE STATE}

Writing down the dynamic problem (9) or rather (11) in the space state allows for the dynamic equations to be uncoupled, even in the presence of non-proportional damping. As is known eigenvalues and eigenvectors of the matrices $\left(\mathbf{A}+\mathbf{B}_{\mathbf{u}} \mathbf{K}_{\mathbf{1}}\right)$ and $\mathbf{A}$ represent the poles (i.e. modal circular frequency and damping) and the modal forms of the controlled or uncontrolled system.

In particular, for an $N$-degrees of freedom system, which is not over-damped, there exist $N$ complex conjugate couples of poles $\lambda_{n}$ :

$$
\lambda_{n}=-\xi_{n} \omega_{n}-i \omega_{n} \sqrt{1-\xi_{n}^{2}} \text { and } \bar{\lambda}_{n}=-\xi_{n} \omega_{n}+i \omega_{n} \sqrt{1-\xi_{n}^{2}}
$$

In (12) $\omega_{n}$ and $\xi_{n}$ represent respectively the vibration circular frequency and the damping factor, both of which are related to the complex conjugate couples of the modal forms $\left(\boldsymbol{\Phi}_{n}, \overline{\mathbf{\Phi}}_{n}\right)$ and assessed by:

$$
\omega_{n}=\sqrt{\operatorname{Re}\left(\lambda_{n}\right)^{2}+\operatorname{Im}\left(\lambda_{n}\right)^{2}} \text { and } \xi_{n}=\frac{-\operatorname{Re}\left(\lambda_{n}\right)}{\sqrt{\operatorname{Re}\left(\lambda_{n}\right)^{2}+\operatorname{Im}\left(\lambda_{n}\right)^{2}}}
$$

By noting the modal properties, the state vector can be written as:

$$
\mathbf{Z}=\sum_{i=1}^{N}\left(p_{i} \boldsymbol{\Phi}_{i}+\bar{p}_{i} \overline{\boldsymbol{\Phi}}_{i}\right)
$$

with $p_{i}$ the $i$-nth principal coordinate. From (14) and (9), taking into account the orthogonality of modal forms $\left(\boldsymbol{\Phi}_{n}, \overline{\boldsymbol{\Phi}}_{n}\right)$ with respect to the state matrix, system (9) or rather (11), uncouples in $N$ complex conjugate couples of first order differential equations.

$$
\dot{p}_{n}+\lambda_{n} p_{n}=\Gamma_{n} \ddot{u}_{g}(t)
$$

in which $\Gamma_{n}$ represents the participation factor of the $n$-nth mode which, in the case of the uncontrolled system is written as:

$$
\Gamma_{n}=\boldsymbol{\Phi}_{\mathbf{n}}^{-1} \mathbf{B}_{\mathrm{g}} \text { for } n=1,2,3 \ldots, 2 N
$$

The dynamic modal properties of the controlled system depend on the following set of parameters:

- $\quad$ the translation vibration period of the system, $T$;

- $\quad$ the supplemental damping, $\xi_{\text {ext }}$;

- $\quad$ the structural eccentricity, $\varepsilon=e / l$;

- $\quad$ the spreads of mass and stiffness about their distribution centroids, $\lambda_{M}=\rho_{M} / l$ e $\lambda_{K}=\rho_{K} / l$;

- $\quad$ the supplemental damping eccentricity, $\varepsilon_{C}=e_{C} / l$;

- the spread of damping about its centroid, $\lambda_{C}=\rho_{C} / l$.

With the aim of evaluating the effect of such parameters, in a wide range, on the dynamic characteristics of the system, a numerical analysis has been carried out. Main results are summarized in figures 3-11, where modal property values of the controlled system are plotted on varying the plan-wise distribution of 
supplemental damping, having fixed $T_{y}=0,1 \mathrm{sec}\left(\omega_{y}=62,8 \mathrm{rad} / \mathrm{sec}\right), \quad \varepsilon=0,2, \quad \lambda_{M}=\lambda_{K}=0,5$, $\xi_{\text {ext }}=0,1$. Figures refer to systems with a coupling effect between the lateral and torsional motion $\left(\lambda_{M}=\lambda_{K}\right)$.

The results show how a plan-wise arrangement of dissipation resources significantly influences two modal properties: the participation factor (fig. 3) and the modal damping (fig. 7). In particular, from figure 3 it is possible to verify the supplemental damping centre as progressively moving towards the mass centre as the participation factor of the second modal form increases, while the same for the first mode is substantially constant. Spread of the damping resources appears to have in this case a secondary role on modal participation factors.

From figure 7, we can see that a bordering of the supplemental damping centroid to the mass one consists in a reduction on the second modal form damping and a correspondent increase of that on the first form. It can be also noted that the greater the spread of the extra-structural dissipation devices, the higher the modal damping on both modal forms. Furthermore, the two modal forms have the same modal damping for a supplemental damping eccentricity value equal to 0.1 , that is in the case in which the damping centre has the same distance from the stiffness and mass centroids.

As a general result, it can be proof that in the cases of lateral and torsional motion coupling $\left(\lambda_{M}=\lambda_{K}\right)$, the modal damping values are symmetrical with respect to a vertical axis passing through the middle point of the segment between the mass and stiffness centroids (figs. 7, 9, 11).

In all the cases analysed, a substantial independence of the modal circular frequency values from the planwise arrangement of supplemental damping has been observed (fig. 4). In addition, it is possible to notice how, in the case of motion coupling, the torsional component is the largest in both the modal forms (figs. $5,6)$.

Furthermore, the numerical parametric analysis allowed for an investigation of the effectiveness of the supplemental dissipation devices on varying the dynamic parameters of the system. The main results are listed as follows:

- the influence of supplemental damping arrangement on modal participation factor appears to decrease when structural eccentricity increases (fig. 8);

- increasing structural eccentricity leads to a simple translation of the above mentioned symmetry axis toward higher values of the supplemental damping eccentricity (fig. 9);

- the increase of the supplemental damping resource accentuates the dependence of the participation factor on the plan-wise distribution (fig. 10);

- modal damping increases adding supplemental dissipation resources (fig. 11);

- other modal properties present a negligible dependence on the investigated parameters.

It is opportune to underline that, being $\rho_{C}$ physically viewable, for classical architectural plan arrangement, as the minimum distance between the supplemental damping centre and the farthest device, and considering the definition of the parameter $l$, the following constraint comes out:

$$
\rho_{C}+e_{C} \leq l
$$

Equation (16) can be written in a non-dimensional form as:

$$
\lambda_{C}+\varepsilon_{C} \leq 1
$$




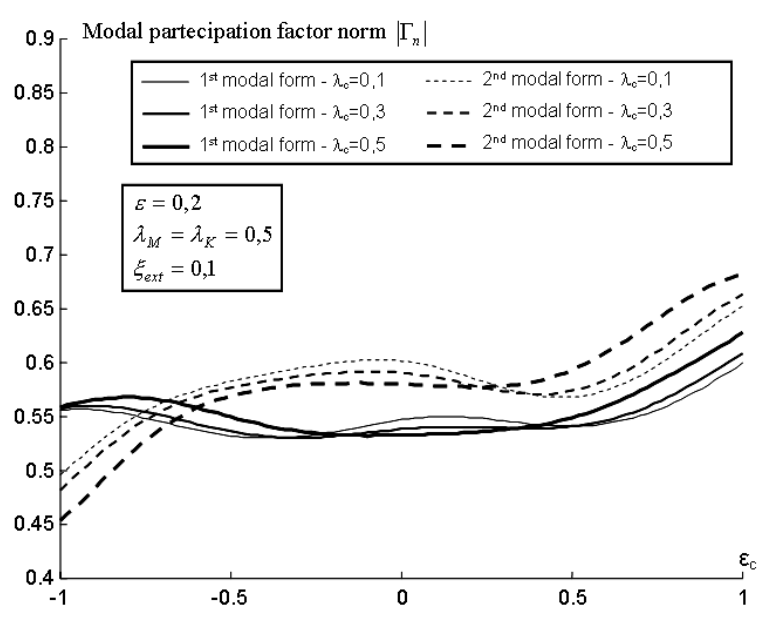

Figure 3: Modal participation factor norm on varying plan-wise distribution of supplemental damping $-T_{y}=0.1 \mathrm{sec}$

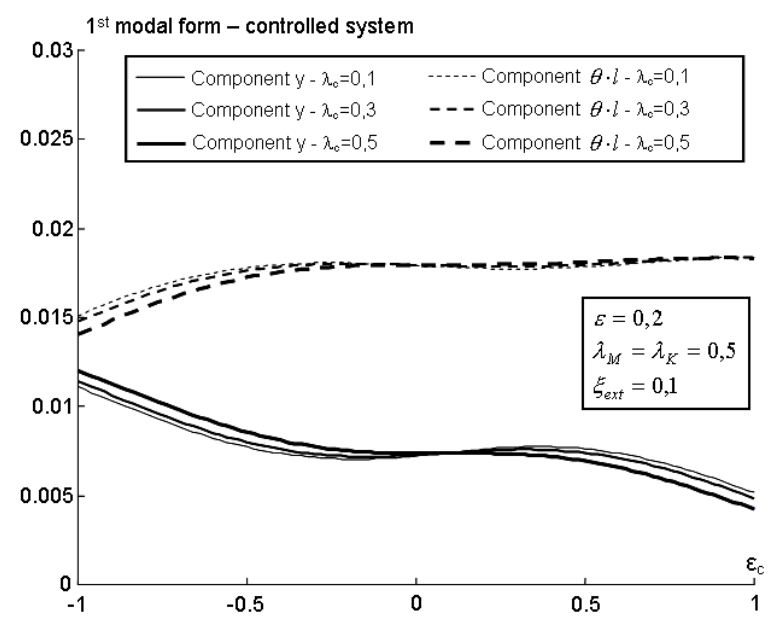

Figure 5: First modal form components on varying plan-wise distribution of supplemental damping $-T_{y}=0.1 \mathrm{sec}$

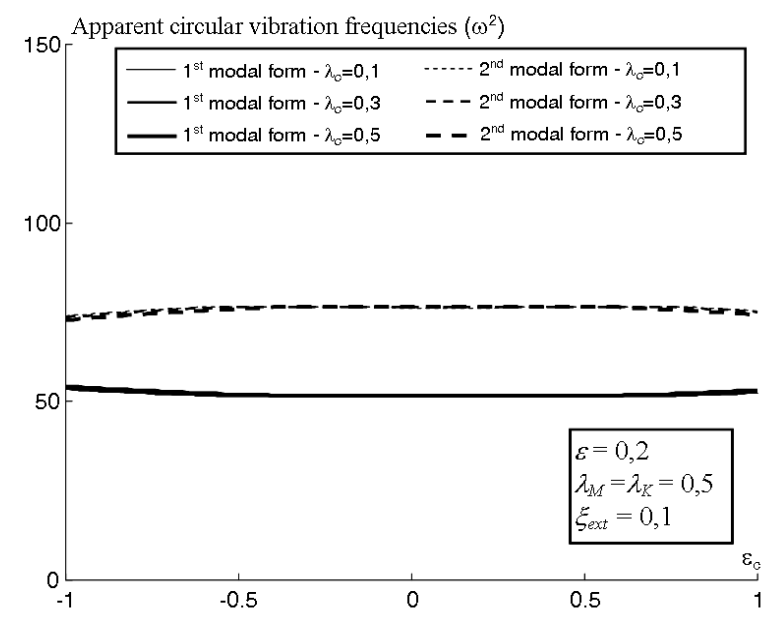

Figure 4: Apparent vibration circular frequencies on varying plan-wise distribution of supplemental damping $-T_{y}=0.1 \mathrm{sec}$

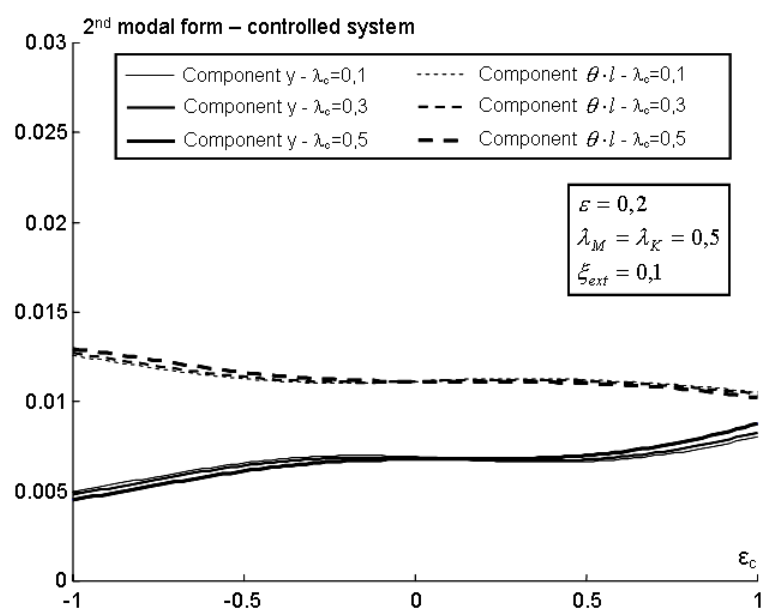

Figure 6: Second modal form components on varying planwise distribution of supplemental damping $-T_{y}=0.1 \mathrm{sec}$

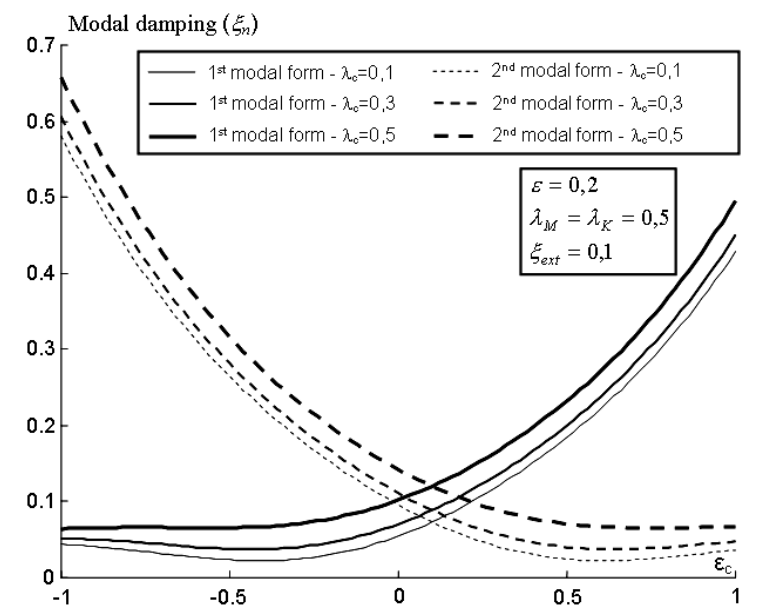

Figure 7: Modal damping on varying plan-wise distribution of supplemental damping $-T_{y}=0.1 \mathrm{sec}$

As far as the control of the lateral-torsional seismic response of the system is concerned, the modal analyses carried out only provide a set of preliminary provisions. 
First of all, no position exists for the supplemental damping centroid to minimize the contribution of both the modal forms. The higher the damping related to the first modal form, the lower is that related to the second modal form, and vice versa. Therefore, the problem of the optimal allocation of dampers is not trivial, and should be faced by means of analytical tools able to synthesize in a performance index the overall modal properties contributing to the seismic response of an asymmetric-plan system.

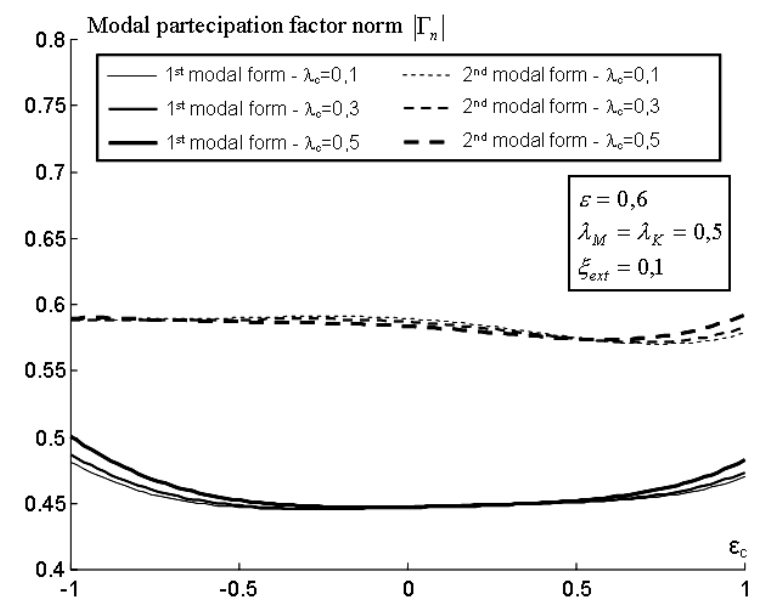

Figure 8: Modal participation factor norm on varying plan-wise distribution of supplemental damping $-T_{y}=0.1 \mathrm{sec}$

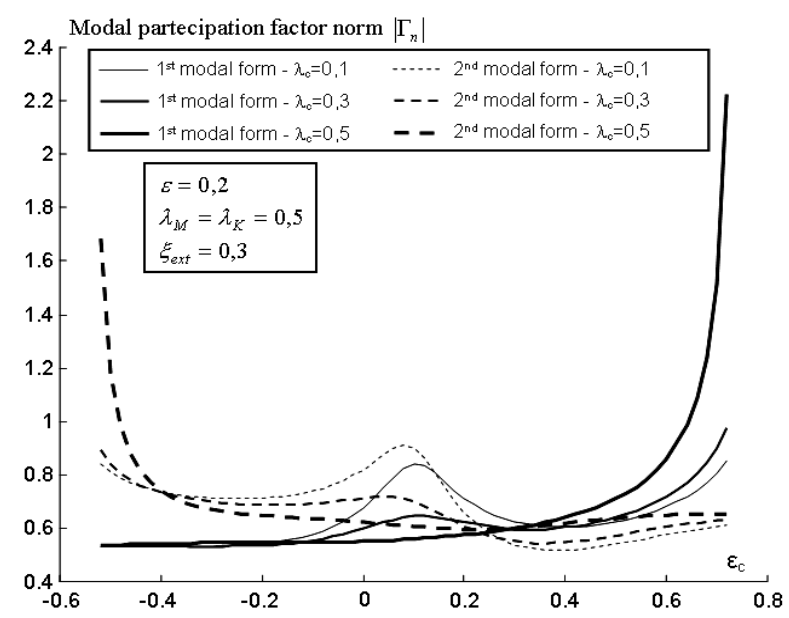

Figure 10: Modal participation factor norm on varying planwise distribution of supplemental damping $-T_{y}=0.1 \mathrm{sec}$

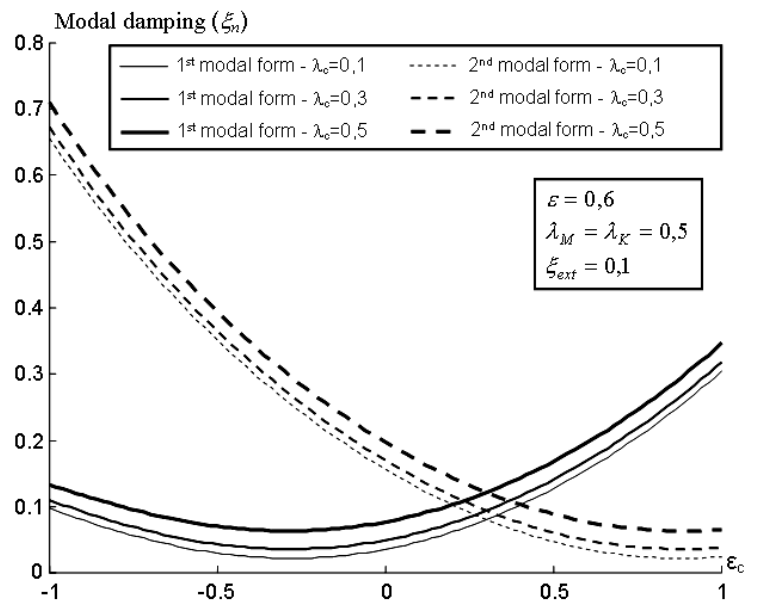

Figure 9: Modal damping on varying plan-wise distribution of supplemental damping $-T_{y}=0.1 \mathrm{sec}$

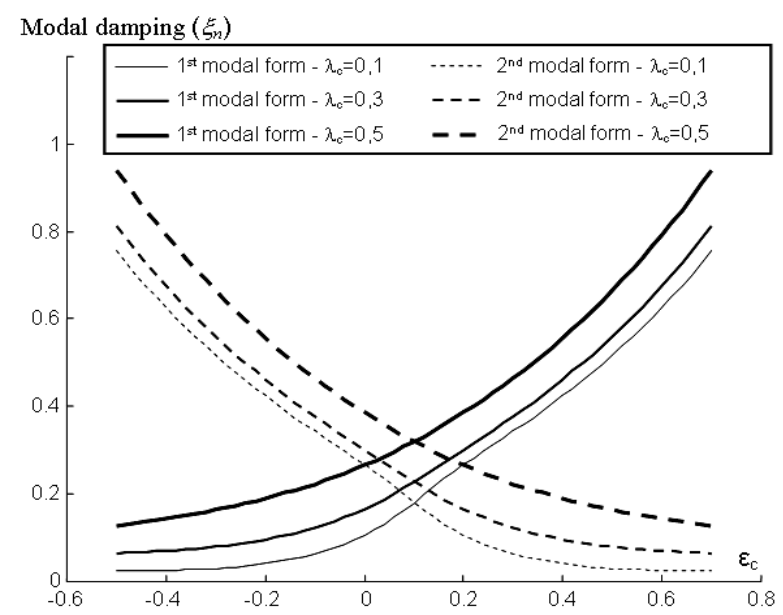

Figure 11: Modal damping on varying plan-wise distribution of supplemental damping $-T_{y}=0.1 \mathrm{sec}$

\section{OPTIMAL PLAN-WISE DISTRIBUTION PROBLEM FOR SUPPLEMENTAL DAMPING}

This work presents a new approach to solving the optimal plan-wise distribution problem of supplemental damping. In particular, such an approach is based on the study of the transfer function relating the maximum edge displacement of the asymmetric-plan system to the input seismic excitation. The evaluation of the $\mathrm{H}_{\infty}$ and $\mathrm{H}_{2}$ norms of such a transfer function represent suitable perfomance index for the definition of optimal design criteria for the plan-wise distribution of extra-structural dampers. 


\section{Transfer function of controlled systems}

Let us consider the Laplace transform of the controlled system. In equation (11) it is possible to evaluate the system's state transform using the following equation:

$$
\mathbf{Z}=\left(s \mathbf{I}-\mathbf{A}-\mathbf{B}_{\mathbf{u}} \mathbf{K}_{\mathbf{1}}\right)^{-1} \mathbf{B}_{\mathbf{g}} s^{2} U_{g}
$$

where $\mathbf{G}(s)=\left(s \mathbf{I}-\mathbf{A}-\mathbf{B}_{\mathbf{u}} \mathbf{K}_{\mathbf{1}}\right)^{-1} \mathbf{B}_{\mathbf{g}} s^{2}$ represents the transfer function vector relating the state of the controlled system to the input seismic excitation. Above all, since our interest lies in investigating the seismic response in terms of edge displacements, we define the transfer functions relating the flexible edge displacement $G^{+}(s)$, and to stiff edge displacement $G^{-}(s)$ to the ground motion as follows:

$$
\begin{gathered}
G^{+}(s)=\left[\begin{array}{llll}
1 & 1 & 0 & 0
\end{array}\right] \cdot \mathbf{G}(s) \\
G^{-}(s)=\left[\begin{array}{llll}
1 & -1 & 0 & 0
\end{array}\right] \cdot \mathbf{G}(s)
\end{gathered}
$$

The plan-wise distribution optimisation has been investigated through the analysis of performance indices defined by the $H_{\infty}$ and $H_{2}$ norms of the transfer functions (20) and (21).

\section{Control in norm $\mathrm{H}_{2}$}

The $H_{2}$ norm of a transfer function $\mathbf{G}(s)$ is defined as (Boyd et al., 1991):

$$
H_{2}=\sqrt{\operatorname{tr}\left(\frac{1}{2 \pi} \int_{-\infty}^{\infty} \mathbf{G}(i \omega) \overline{\mathbf{G}}(i \omega) d \omega\right)}
$$

where the symbols $\operatorname{tr}$ and $\bar{*}$ respectively represent the trace and the transpose complex conjugate operators. If one considers that the power density spectral response of the system $\mathbf{S}_{\text {out }}(\omega)$ to a white noise, characterized by $\mathbf{S}_{i n}(\omega)=1$, is given by

$$
\mathbf{S}_{\text {out }}(\omega)=\mathbf{G}(i \omega) \overline{\mathbf{G}}(i \omega)
$$

the equation (23) shows that norm $\mathrm{H}_{2}$ represents a measure of the root mean square (RMS) of the system response to white noise. Therefore, regulating the system response $\mathbf{Z}$, with the target to minimize the $H_{2}$ norm, means to minimize the RMS response. In the time domain the system RMS response is given by:

$$
\sigma_{z}=\sqrt{\lim _{\varepsilon \rightarrow \infty} \frac{1}{2 \tau} \int_{-\varepsilon}^{\varepsilon} \mathbf{z}^{T}(t) \mathbf{z}(t) d t}
$$

the problem of the $\mathrm{H}_{2}$ control can lead back to the minimization of the following performance index:

$$
J_{H_{2}}=\int_{0}^{\infty} \mathbf{z}(\tau)^{T} \mathbf{z}(\tau) d \tau
$$

where it is assumed that the system is at rest until the time $t_{0}=0$. 


\section{Control in norm $H_{\infty}$}

The $H_{\infty}$ norm of a stable system transfer matrix is defined as (Boyd et al., 1991):

$$
H_{\infty}=\sup _{r(s)>0} \sigma[\mathbf{G}(s)]
$$

Where $\sigma($.$) is the maximum singular value operator defined as:$

$$
\sigma(\mathbf{G}(s))=\max _{\mathbf{d} \neq 0} \frac{\mathbf{d}^{*} \mathbf{G}^{*}(s) \mathbf{G}(s) \mathbf{d}}{\mathbf{d}^{*} \mathbf{d}}
$$

Such an expression shows that the $H_{\infty}$ norm represents a measure of the upper extreme of the rms outputinput ratio. Therefore, in this sense we are talking about design control in the "worst case". Consider a linear system, in a null state until $t=0$, described in the time domain by:

$$
\left\{\begin{array}{l}
\dot{\mathbf{x}}=\mathbf{A x}+\mathbf{B u} \\
\mathbf{y}=\mathbf{C x}
\end{array}\right.
$$

Let the gain $L_{2}$ have the following expression:

$$
L_{2}=\sup _{\mathbf{u} \neq 0} \frac{\sqrt{\int_{0}^{\infty}\|\mathbf{y}(t)\|^{2} d t}}{\sqrt{\int_{0}^{\infty}\|\mathbf{u}(t)\|^{2} d t}}
$$

It is possible to demonstrate that $L_{2}$ coincides with the $H_{\infty}$ norm, so such a norm can be physically interpreted as the upper extreme of the $L_{2}$ gain on varying the input signal.

\section{Optimal plan-wise damper distribution}

In figures 12-17, values of the $H_{2}$ and $H_{\infty}$ norms applied to the transfer function $G^{+}(s)$ and $G^{-}(s)$ on varying mechanical parameters describing the plan-wise distribution of the extra-structural damping, $\varepsilon_{C}$ and $\lambda_{C}$, are plotted. In particular, figure 12-13 show the $H_{\infty}$ norm trend for both $G^{+}(s)$ and $G^{-}(s)$ transfer function and for $\varepsilon=0.2-0.4$. In figures 14-17, according to the aim of limiting the maximum of both edge displacements, for every considered $\left(\varepsilon_{C}, \lambda_{C}\right)$ couple, the maximum value of the $H_{\infty}$ (figs. 1415) and $H_{2}$ (figs. 16-17) norm between the two transfer function under consideration is represented.

These results allow for the evaluation of an optimal plan-wise arrangement of the dissipation resource described by the parameters $\left(\varepsilon_{C, \text { opt }}, \lambda_{C, \text { opt }}\right)$, considering also the physical limit expressed by equation (17) (dotted line). Specifically, the dashed line in figures 14-17 represents the optimal value of the damping centre eccentricity having fixed the spread of damping about its centre. This optimal condition is characterized by the equality of the $H_{\infty}$ or $H_{2}$ norms for both the transfer functions $G^{+}(s)$ and $G^{-}(s)$. 

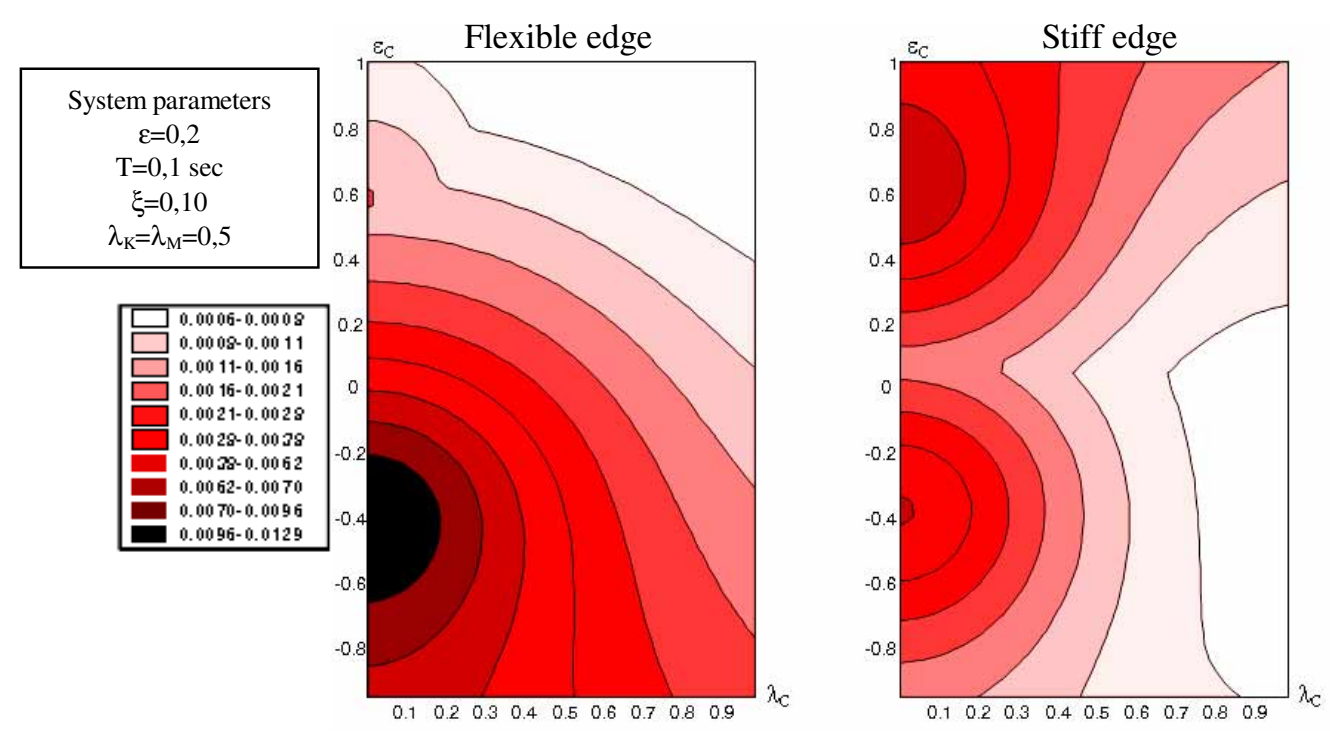

Figure 12: $\mathrm{H}_{\infty}$ norms of the transfer function between seismic input and edge displacement
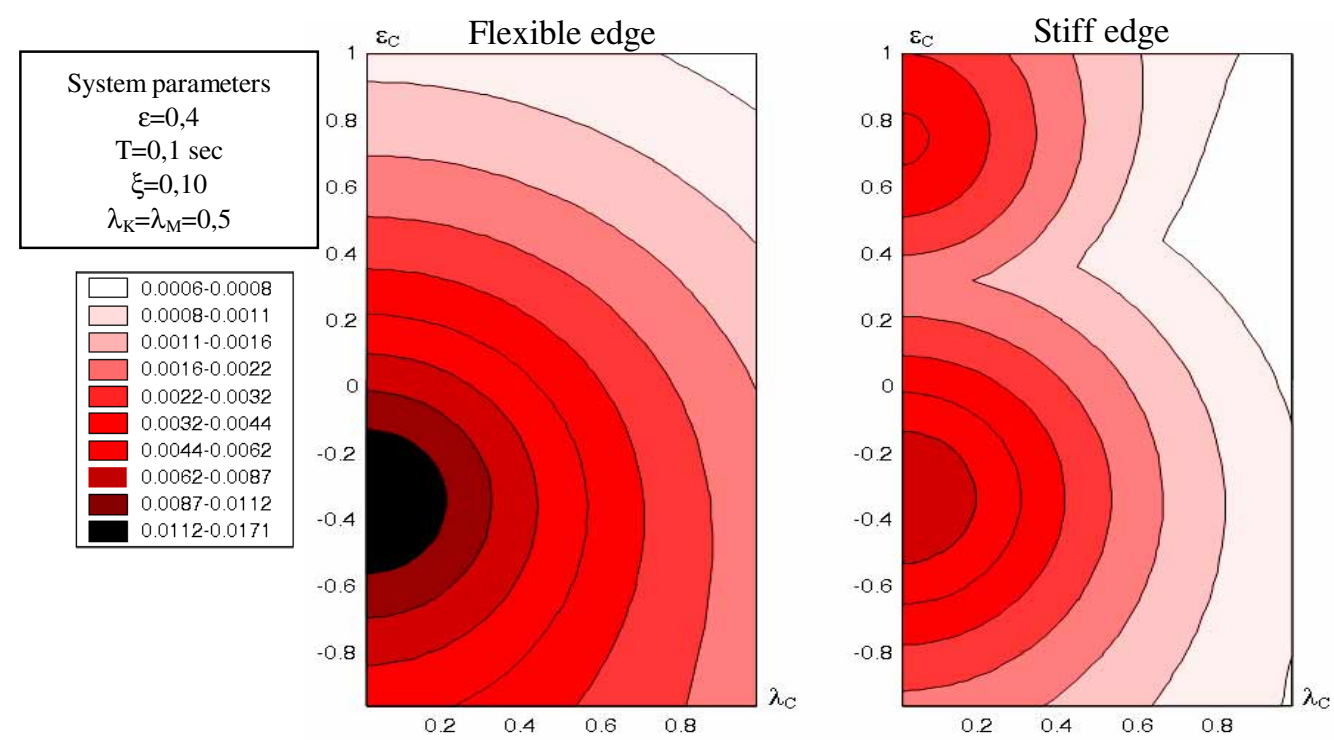

Figure 13: $\mathrm{H}_{\infty}$ norms of the transfer function between seismic input and edge displacement

From the obtained results the following general considerations can be derived:

- the increase of the supplemental damping centre eccentricity toward the flexible edge reduces the flexible edge displacement, but it worsens the response of the stiff one.

- optimal control of stiff edge displacement is obtained when the supplemental damping centre is located between the mass and stiffness centroids (fig. 12-13);

- the increase in the supplemental damping spread has a beneficial effect on the control of both flexible and stiff edge displacements (fig. 12-13);

- the optimal condition, which minimizes both flexible and stiff edge $H_{\infty}$ norm, provides for a allocating of the extra-structural damping centroid in correspondence to the mass centre, in the case of $\lambda_{C}=0$. By considering increasing values for $\lambda_{C}$ the optimal value for $\varepsilon_{C}$ increases too (figs. 14-17); 
- the optimal plan-wise distribution using the $\mathrm{H}_{2}$ norm presents slightly higher values of the supplemental damping centre eccentricity in comparison to the $H_{\infty}$ norm case;

- the increase in structural eccentricity determines a corresponding higher value for the optimal damping eccentricity;

- using a value of the parameter $\lambda_{C}$ that is higher than the optimal value does not significantly affect the $H_{\infty}$ and $H_{2}$ norms.

With reference to the reported cases the supplemental damping optimal distribution parameter dispositions are indicated in table 1:

Table 1: Optimal arrangement of extra-structural dissipation resource

\begin{tabular}{|c|c|c|}
\hline & Norm & Optimal distribution \\
\hline \multirow{2}{*}{$\varepsilon=0,2$} & $H_{\infty}$ & $\varepsilon_{\mathrm{C}}=0.33 \lambda_{\mathrm{C}}=0.67$ \\
\cline { 2 - 3 } & $H_{2}$ & $\varepsilon_{\mathrm{C}}=0.41 \lambda_{\mathrm{C}}=0.59$ \\
\hline \multirow{2}{*}{$\varepsilon=0,4$} & $H_{\infty}$ & $\varepsilon_{\mathrm{C}}=0.57 \lambda_{\mathrm{C}}=0.43$ \\
\cline { 2 - 3 } & $H_{2}$ & $\varepsilon_{\mathrm{C}}=0.62 \lambda_{\mathrm{C}}=0.38$ \\
\hline
\end{tabular}
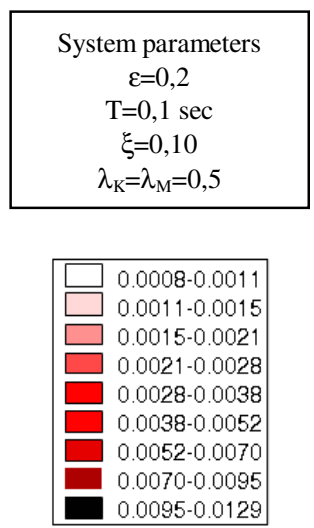
negative edge transfer functions

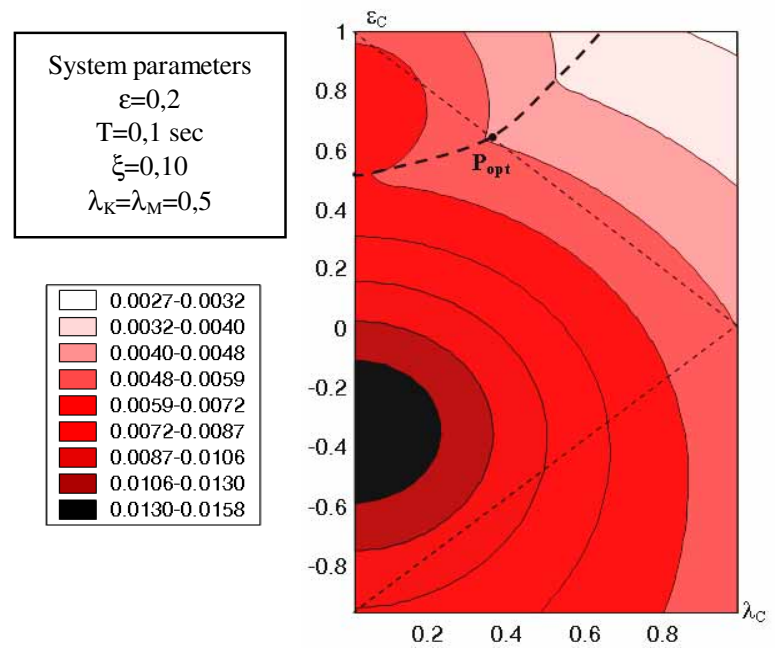

Figure 16: Maximum $\mathrm{H}_{2}$ norm considering both positive and negative edge transfer functions
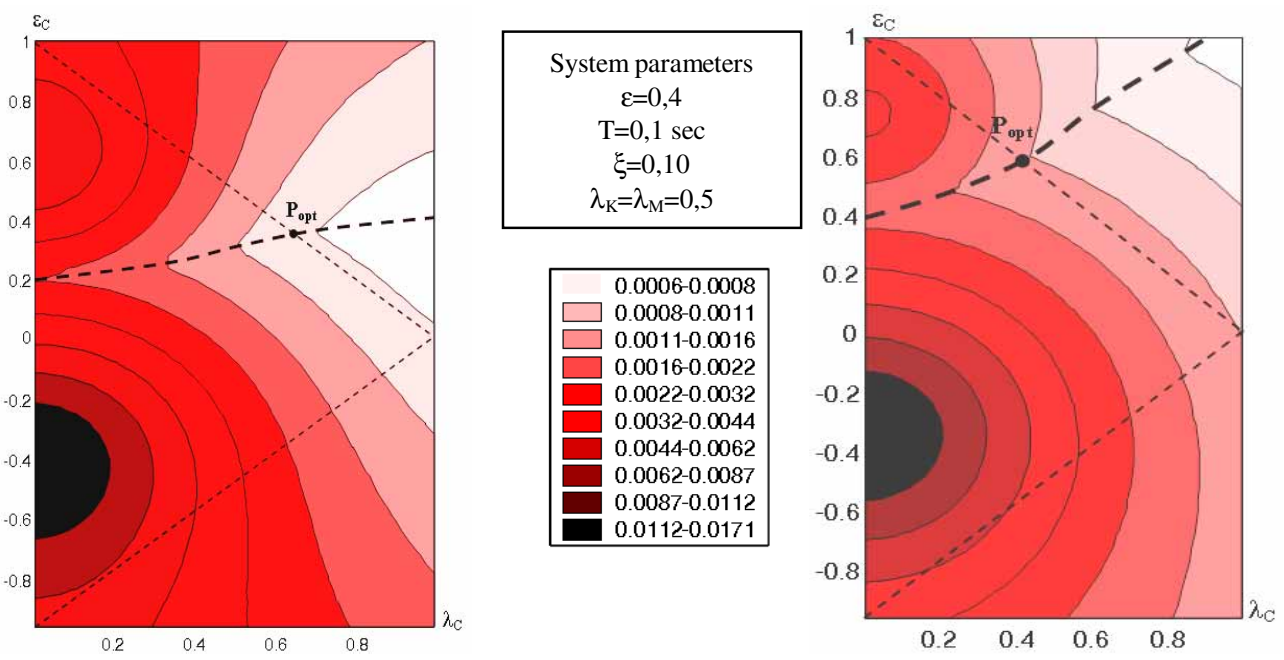

Figure 15: Maximum $\mathrm{H}_{\infty}$ norm considering both positive and negative edge transfer functions

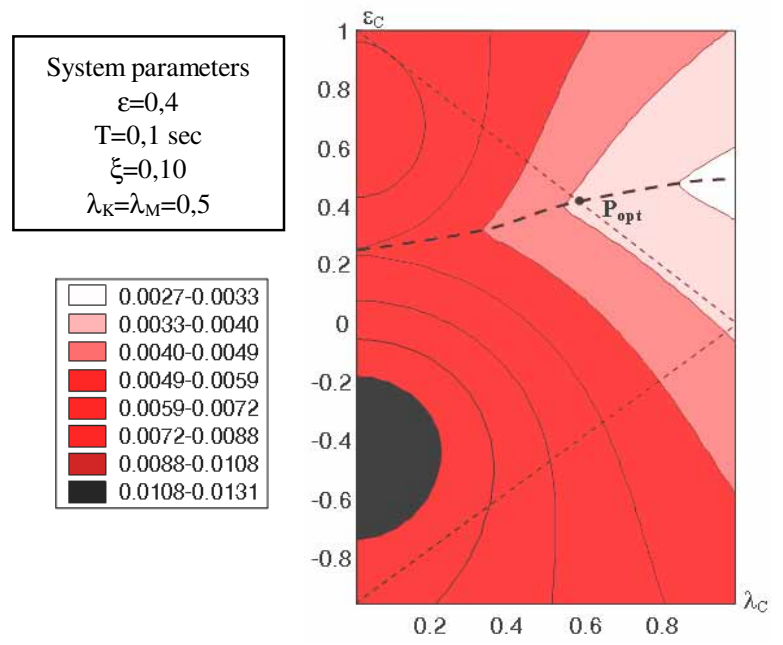

Figure 17: Maximum $\mathrm{H}_{2}$ norm considering both positive and negative edge transfer functions 


\section{NUMERICAL TESTS}

In order to validate the achieved results, a wide numerical tests to evaluate the dynamic response of a 2DOF system $(y, \theta)$ in the case of seismic excitations having only one translation component are carried out. Several plan-wise distributions of supplemental damping are investigated and compared with the optimal distributions listed in table 1. In particular, the mean value of the maximum edge displacement response of the systems is evaluated for 10 synthetic seismic excitations compatible with response spectra class A described in EuroCode 8 (prEN 1998-1:2003). Two different vibration periods, selected in the circular frequency range in which the synthetic seismic inputs present high energetic content, and different supplemental damping values are considered. The results are summarized in the following tables 2-3.

Results confirm the key role of an optimal plan-wise distribution of supplemental damping. It is possible to note, indeed, how equal amounts of dissipation resources can be consistent with maximum edge displacement different for a factor equal to 4. Furthermore, as concerns maximum displacement control, an incorrect disposition of supplemental damping lead to the dissipation resources be ineffective. It also serves to emphasize, as the results in tables 2-3 show, the coherence of the $H_{\infty}$ and $H_{2}$ norms as a mathematical tool to investigate the optimal distribution of dampers.

The optimal value of distribution parameters $\left(\varepsilon_{C, \text { opt }}, \lambda_{C, \text { opt }}\right)$ allows for a reduction of edge maximum displacement in comparison with all other considered plan-wise arrangements of dampers, especially for $\mathrm{T}=0,25 \mathrm{sec}$.

Finally, the seismic response of structures with a high vibration period to the recorded seismic event of Mexico City (1985) have been investigated. This specific seismic excitation is particularly disadvantageous for flexible systems, therefore the dynamic response of the controlled system allows us to verify the robustness of the proposed control strategy. Results are presented in table 4, while in figs. 18-19 time-histories for flexible edge displacement are plotted, to compare the seismic response for correct and incorrect plan-wise distribution of the dissipative resource. Also in this case, the results obtained confirm the effectiveness of the proposed approach, in particular for high values of extra-structural damping and structural eccentricity.

Table 2: Maximum edge displacement mean values [m]

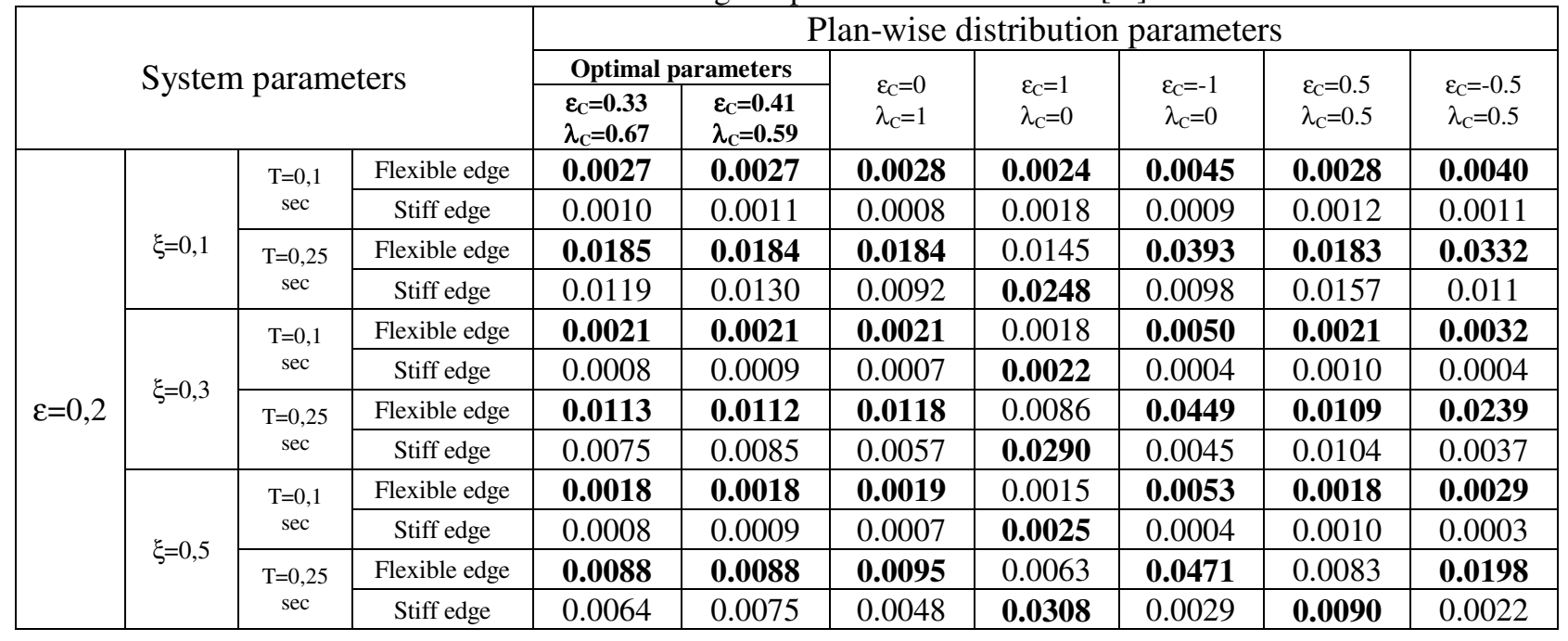


Table 3: Maximum edge displacement mean values [m]

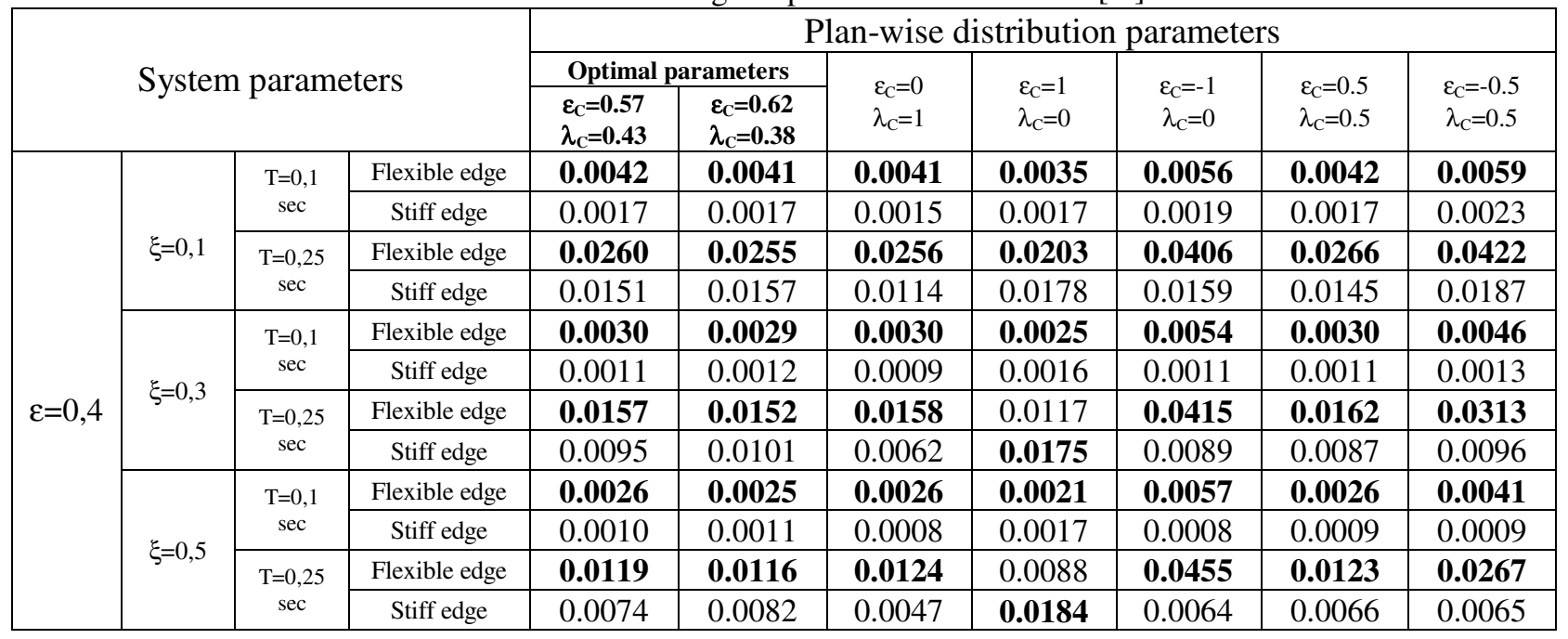

Table 4: Maximum edge displacement mean values [m] - Mexico City event

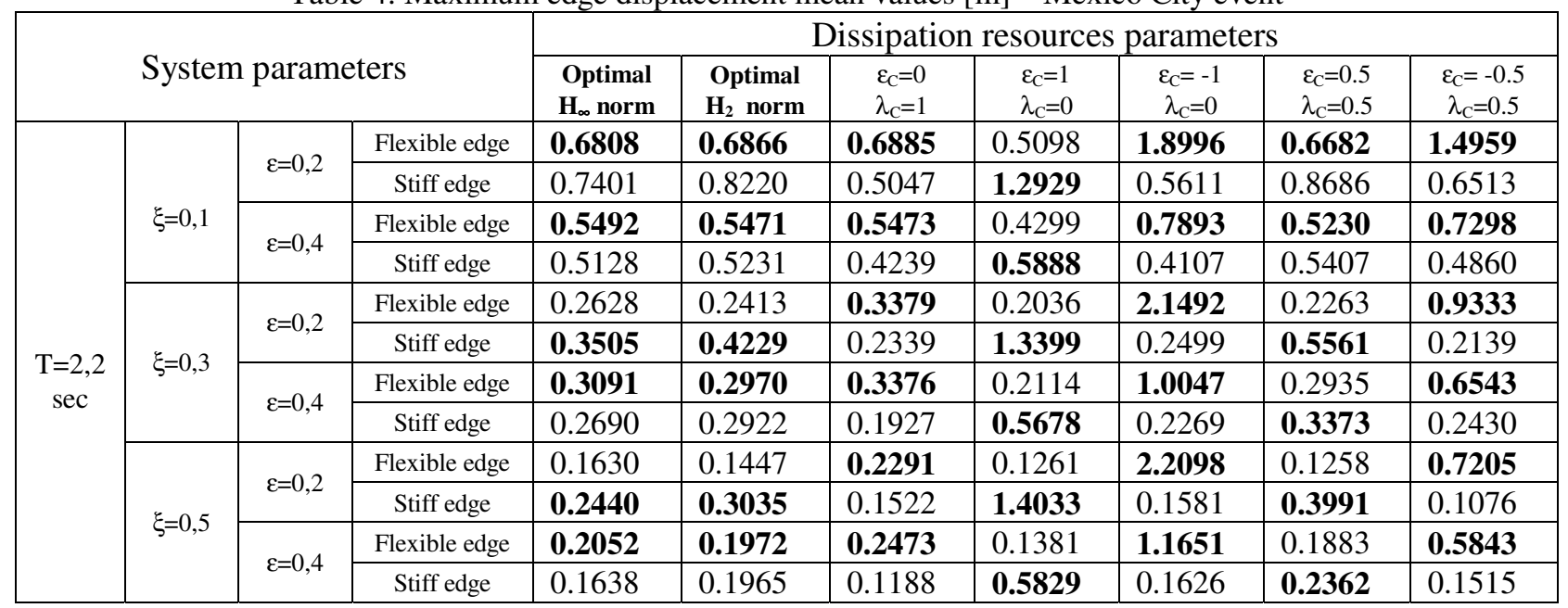

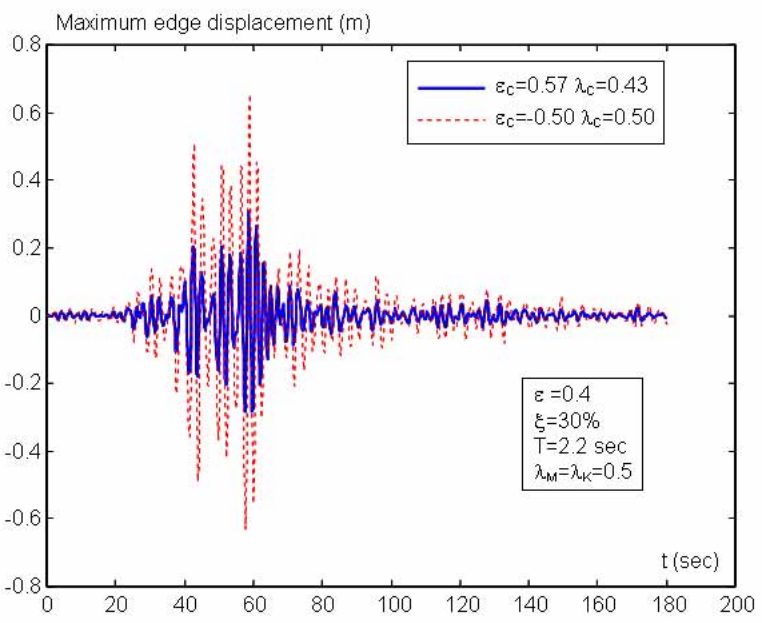

Figure 18: Maximum edge displacement comparison - Mexico City seismic event

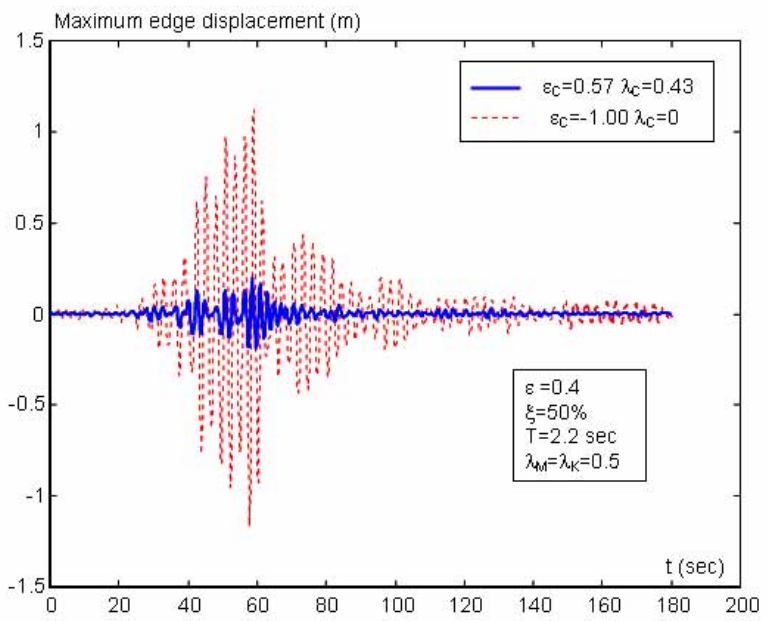

Figure 19: Maximum edge displacement comparison - Mexico City seismic event 


\section{CONCLUSION}

A new approach for defining plan-wise optimal arrangement of supplemental damping in asymmetric-plan systems has been carried out. The dynamic problem has been investigated in the state space representation showing that the supplemental dissipation resources work as a closed-loop feedback control action. This allowed for a better physical understanding of the problem and for the formulation of optimal plan-wise design criteria for additional damping devices showing that moving the damping centre through the flexible edge lead to swap the stiff role between the edges.

Optimal plan-wise arrangement of supplemental damping, obtained by using $H_{\infty}$ and $H_{2}$ norms, take place when supplemental damping centre is close to the mass center for low value of damping radius of gyration, while as this radius increases, the optimal location moves through the flexible edge.

Such design provisions have been positively verified through wide numerical tests comparing the seismic response to synthetic and real excitations for optimal and non-optimal plan-wise distributions of supplemental damping. Such numerical analyses have also shown how an no optimal arrangement of extra-structural dissipation devices can result in maximum edge displacement four times greater than that which may be achieved through adopting the optimal solution.

\section{ACKNOWLEDGMENTS}

This work is part of the project of the Campania Regional Center of Competence "Analysis and Monitoring of the Environmental Risk" supported by the European Community on Provision 3.16.

\section{REFERENCES}

1. Doyle J.C., Glover K., Khargonekar P., Francis B., State-space solution to standart $\mathrm{H}_{2}$ and $\mathrm{H}_{\infty}$ control problems. IEEE Transaction on Automatic Control, 34, 831-847, 1989.

2. Eurocode 8, Design of structures for earthquake resistance - Part 1: General rules, seismic actions and rules for buildings, ENV 1998-1:2003, 2003.

3. Goel RK, Effects of supplemental viscous damping on seismic response of asymmetric-plan system. Earthquake Engineering and Structural Dynamics 1998; 27:125-141

4. Goel RK, Seismic behaviour of asymmetric-plan buildings with supplemental damping. Earthquake Engineering and Structural Dynamics 2000; 29:461-480

5. Goel RK, Booker CA, Inelastic seismic response of asymmetric systems. Earthquake Engineering and Structural Dynamics 2001; 30:411-430

6. Hejal, R. and Chopra, A., Earthquake response of torsionally-coupled buildings, Report UCB/EERC-87/20, December 1987

7. Kan, C. L. and Chopra, A. K., Linear and nonlinear earthquake responses of simple torsionally coupled systems, Report UCB/EERC-79/03, February 1979

8. Kreyszig E., Advanced Engineering Mathematics ( $7^{\text {th }}$ edition), Wiley, New York, 1993

9. Lin W-H, Chopra AK, Understanding and predicting effects of supplemental viscous damping on seismic response of asymmetric one-storey systems. Earthquake Engineering and Structural Dynamics 2001; 30:1475-1494

10. Palazzo B., Petti L., Aspects of structural vibration passive control, International Journal of MECCANICA, Vol. 32 N.6 December 1997

11. Pekau OA, Guimond R., Controlling seismic response of eccentric structures by friction dampers, Earthquake Engineering and Structural Dynamics, 1991; 20(6):505-52 